\title{
Um estudo dos relatos afetivos subjetivos a estímulos do International Affective Picture System em uma amostra geriátrica brasileira
}

\author{
Subjective affective ratings to photographic stimuli of the International Affective Picture \\ System in a Brazilian elderly sample
}

\author{
Weyler Galvão Pôrto ${ }^{1}$, Paulo Bertolucci ${ }^{2}$, Rafaela Larsen Ribeiro ${ }^{3}$, Orlando Francisco Amodeo Bueno \\ ${ }^{1}$ Médico. Mestre em Gerontologia. Doutor em Psicobiologia. Coordenador, Ambulatório de Distúrbios da Memória, Comportamento e Humor, Setor de \\ Geriatria, Hospital Estadual Mário Covas, Fundação do ABC, Santo André, SP. ${ }^{2}$ Doutor. Chefe, Seção de Neurologia Comportamental, Universidade \\ Federal de São Paulo (UNIFESP), São Paulo, SP. ${ }^{3}$ Doutora. Professora adjunta visitante, Departamento de Psicobiologia, UNIFESP. ${ }^{4}$ Livre-docente. \\ Professor associado livre-docente, Departamento de Psicobiologia, UNIFESP.
}

\section{Resumo}

Introdução: A literatura científica indica a possibilidade de a percepção da emoção e a formação da memória emocional serem discordantes entre jovens e idosos. A mesma é pobre ao explorar essa possibilidade. Neste estudo, relatamos os resultados obtidos em um experimentopiloto com uma amostra de idosos brasileiros, que classificaram subjetivamente, através da escala Self Assessment Manikin, imagens oriundas do International Affective Picture System.

Método: Quarenta e oito idosos voluntários da Universidade Aberta da Terceira Idade, saudáveis clínica e cognitivamente, avaliaram o caráter alertante e a valência afetiva de 71 imagens do International Affective Picture System, aleatoriamente escolhidas.

Resultados: $\mathrm{O}$ grau de alerta reportado por idosos diante de um estímulo emocional é tanto maior quanto menor o prazer provocado por essa imagem-estímulo, resultando na existência de uma forte correlação negativa $(\mathrm{r}=0,93)$ entre o grau de alerta e o estímulo desprazeroso. Em uma comparação do acima obtido com outro experimento normativo semelhante feito com jovens brasileiros e americanos, apontou-se para uma possível diferença cultural na forma de relatar subjetivamente um estímulo emocional.

Conclusões: Os resultados obtidos com esta amostra estudada sugerem que pode existir uma diferença nos relatos afetivos entre os jovens e idosos, onde uma normatização do International Affective Picture System para uma amostra maior, representativa da população de idosos, seria útil para responder esta questão.

Descritores: International Affective Picture System, Self Assessment Manikin, idosos, Brasil.

\begin{abstract}
Introduction: The scientific literature points to a possible bias in the form perception and emotional memory are constructed when elderly and young individuals are compared. However, this possibility is underexplored. This paper presents the results obtained from a pilot study based on an elderly emotional subjective report after evaluation using the International Affective Picture System images and Self Assessment Manikin scale scores.

Method: Forty-eight clinically and cognitively capable elderly volunteer subjects from the Third Age Open University evaluated 71 randomly chosen images of the International Affective Picture System in terms of arousal and affective valence.

Results: For the elderly, the greater the arousal, the smaller the pleasure resulting in a strong negative correlation $(r=0.93)$ observed between arousal and negative valence. A comparison with another similar normative experiment performed in young Brazilian and American individuals showed a possible cultural difference in subjective reports of emotional stimuli.

Conclusions: This investigation indicates that there may be a difference between elderly and young individuals when affective reports of arousal are studied. A normalization of the International Affective Picture System for the elderly in a larger sample, representative of the population, might be useful to address this issue.
\end{abstract}

Keywords: International Affective Picture System, Self Assessment Manikin, elderly, Brazil. 


\section{Introdução}

As revoluções epidemiológica e demográfica que culminaram com o envelhecimento populacional humano introduziram novos paradigmas nas neurociências. Até aqui, a maioria dos trabalhos científicos envolvia somente jovens e crianças como objeto de estudo. No entanto, especula-se que nem todos os resultados, materiais e métodos obtidos dos estudos com jovens podem ser extrapolados diretamente para a geriatria e gerontologia sem prévias adaptações às necessidades dos idosos ${ }^{1-5}$. Em geral, a ciência é pobre na exploração desse tema, e a situação fica ainda mais complexa quando se refere à memória emocional dessa fração demográfica ${ }^{2,5}$.

O envelhecimento, em geral, é associado a um período de declínio na evolução humana. Os idosos podem apresentar déficits cognitivos como um resultado de mudanças fisiológicas que estão intrinsecamente ligadas ao processo de envelhecimento; doenças sistêmicas que comprometem o sistema nervoso central (SNC); e também doenças primárias do sistema nervoso autônomo, periférico e central. No entanto, existe um grupo de indivíduos que não se enquadra nas três categorias citadas e envelhecem com sucesso. Estes são considerados saudáveis e totalmente inseridos em seus contextos sociais. Mesmo assim, podemos observar um declínio cognitivo primário associado com o envelhecimento (DCPRE). O DCPRE é caracterizado por redução da velocidade de processamento, declínio na inteligência fluida e déficit de memória episódica ${ }^{1,6}$. Esses sintomas podem ser responsáveis por erros cognitivos comumente feitos por indivíduos idosos. Entre eles, podemos mencionar o reconhecimento inadequado de palavras semanticamente relacionadas e figuras de categorias semelhantes; capacidade diminuída para discriminar novos e velhos detalhes em tarefas de recordação livre; confusões de situações estereotipadas como parte de outra apresentada anteriormente; e também erro na atribuição de fonte após uma dada informação ${ }^{7}$.

Por outro lado, também se questiona qual o efeito do nível de alerta entre as características do DCPRE - e mesmo seu efeito na memória declarativa de idosos ditos saudáveis. Mais freqüentes ainda são as dúvidas sobre a memória emocional de sujeitos idosos. Nessa linha, alguns autores têm mostrado que quando os idosos são comparados com os jovens, a) adquirem controle e regulação sobre seu bem-estar emocional, b) as emoções ganham centralidade no processo cognitivo, e c) apresentam relativa preservação de áreas anatômicas envolvidas no processo de alerta. Aparentemente, portanto, nem tudo é declínio em cognição e emoção entre os idosos, e esse fato deve ser mais adequadamente explorado em estudos controlados sobre emoção e envelhecimento ${ }^{4,5,8}$.
As emoções podem ser consideradas fenômenos biológicos mensuráveis. Em geral, os fenômenos emocionais podem ser expressos e então medidos em três categorias: 1) comportamentos emocionais funcionais apresentados sob a forma de atos e seqüências comportamentais; 2) linguagem emocional; e 3) reações fisiológicas ${ }^{9-12}$.

Correntemente, o método perfeito passível de replicar as emoções em estudos controlados, exatamente como ocorre na vida real, está longe do que podemos sonhar como padrão-ouro. As metodologias que provocam medo e alerta em indivíduos idosos são limitadas por aspectos éticos óbvios. Para esse propósito, normalmente podemos utilizar a busca por memórias autobiográficas (conscientes ou induzidas por hipnose), situaçães induzidas de humor e/ou emoção ou recriação de ambientes fóbicos através de fotos, vídeos ou palavras ${ }^{13,14}$. O International Affective Picture System (IAPS) ${ }^{15}$, bem como o International Affective Digitized Sound System (IADS) e o Affective Lexicon of English Words (ANEW), entre outros métodos, visam à recriação de uma atmosfera emocional pelo uso de figuras, sons e/ ou palavras emocionais. A resposta emocional suscitada por esses métodos é medida através de relatos afetivos subjetivos, mensurados em uma escala analógica conhecida como Self Assessment Manikin (SAM), e/ou pode ainda ser objetivamente mensurada através de respostas fisiológicas e comportamentais.

Entre os instrumentos de mensuração apresentados acima, o IAPS apresenta características específicas quando utilizado por diferentes países, como já mostrado em estudos espanhóis, brasileiros e americanos. Em geral, conclui-se que um mesmo estímulo emocional pode ser percebido diferentemente por díspares culturas ${ }^{16,17}$. Nesses mesmos citados trabalhos, existe uma proposta de adaptação do IAPS para cada alvo populacional ou amostra a ser estudada no sentido de prever e evitar eventuais erros de conclusão pré-conceituais. Fatores como idade, doenças, educação, entre outros tantos, podem interferir com a avaliação do estímulo afetivo.

Assim, com o objetivo de explorar a existência de uma possível diferença teórica, como acima exposto, na avaliação de relatos afetivos subjetivos, entre idosos e jovens brasileiros, desenvolvemos este projeto-piloto, utilizando-nos das imagens do IAPS como estímulos emocionais.

\section{Método}

\section{População}

O projeto-piloto em questão foi elaborado pelo Departamento de Psicobiologia e pelo Departamento de Neurologia do Setor de Neurologia Comportamental da 
Universidade Federal de São Paulo (UNIFESP) e realizado com os alunos, de uma forma voluntária, da Universidade Aberta da Terceira Idade (UATI). A UATI apresenta 175 alunos acima de 60 anos de idade regularmente matriculados; $83 \%$ são mulheres, e $17 \%$ são homens. Deste universo, uma amostra de 48 alunos $(27,43 \%)$ foi escolhida por amostragem aleatória simples - 42 mulheres $(87,5 \%)$ e seis homens $(12,5 \%)$-, não diferindo estatisticamente quanto à idade e à escolaridade $-68,65 \pm 6,7$ anos (média \pm DP), 8 anos de escolaridade. Todos os participantes usavam óculos com lentes corretoras.

Todos eles eram independentes para atividades básicas (AVD) e instrumentais (AVDI) da vida diária; $100 \%$ dos indivíduos apresentavam seis pontos possíveis em seis (6/6) para AVD e oito em oito (8/8) para AVDI ${ }^{18}$. Não apresentavam história clínica de neoplasias, distúrbios metabólicos, infecciosos ou psiquiátricos, traumatismo crânio-encefálico, perda de consciência, abuso de drogas ilícitas ou medicamentos que pudessem alterar a cognição. Seus resultados na escala isquêmica de Hachinski foram de $<4^{19}$, e o escore no teste do desenho espontâneo do relógio foi de $12,7 \pm 2,6^{20,21}$.

\section{Material}

Inicialmente, foram selecionados 40 diapositivos, e, posteriormente, mais 71 foram escolhidos do IAPS ${ }^{15}$ por processo aleatório simples.

\section{Procedimento}

Na primeira etapa, os 40 primeiros diapositivos foram escolhidos e divididos em dois subgrupos de 20 imagens. Cada subgrupo foi balanceado quanto à valência afetiva e nível de alerta para que não houvesse extremos afetivos entre os grupos. $\mathrm{O}$ grupo de 48 idosos foi também dividido em dois subgrupos aleatoriamente. Um subgrupo de 20 imagens foi apresentado a 24 idosos (subgrupo A) e pedido para que eles observassem as imagens por 5 segundos e as classificassem em outros 10 segundos de acordo com o manual de aplicação produzido para uma amostra de jovens ${ }^{15,17}$. A outra metade foi apresentada aos demais 24 idosos (subgrupo B).

Em uma segunda etapa, mais 71 diapositivos foram escolhidos de forma aleatória e apresentados de forma balanceada, em grupos de 10 sujeitos/sessão, sendo analisados posteriormente segundo a metodologia apresentada no manual prático de aplicação e na normatização para jovens brasileiros adaptada para pacientes idosos após a etapa anterior. Cada uma das imagens pertencentes ao IAPS recebeu um escore SAM relativo às dimensões valência afetiva e nível de alerta, determinado por cada participante do estudo. A média desses escores determina o valor afetivo subjetivo de cada imagem relatado pelos idosos. Os valores obtidos do SAM foram então comparados com seus correspondentes obtidos de estudos com indivíduos jovens.

No início de cada sessão, os sujeitos assinavam o Consentimento Livre e Esclarecido aprovado pelo Comitê de Ética em Pesquisa da UNIFESP, conforme resolução CEP $n^{\circ}$ 438/01, de 11 de junho de 2001, sendo, nesta hora, esclarecidas as dúvidas pelo pesquisador.

\section{Estatística}

O programa Statistica (versão 5, 1997) foi utilizado para analisar estatisticamente todo o material e dados do trabalho. Arbitrariamente, foi definido que os escores do SAM para o propósito de cálculos teriam os seguintes valores: prazer: 6,01-9; neutro: 3,01-6; desprazer: 1-3; alerta: 6,01-9; relaxamento: 1-3.

As unidades mensuráveis e avaliadas na análise estatística foram as médias das médias alcançadas em cada grupo e entre eles. Os grupos não apresentaram uma curva normal estatística nem homocedasticidade. Portanto, foi utilizada metodologia não-paramétrica para a análise de variância - testes de Kruskal-Wallis, teste U de Mann-Whitney e coeficiente de correlação de Spearman $\left(\mathrm{r}_{\mathrm{s}}\right)$. O nível de significância foi de $\alpha_{2}=0,05$.

\section{Resultados}

A primeira etapa foi utilizada para adaptarmos a versão lápis-e-papel do IAPS ${ }^{15}$ às necessidades dos idosos. Observamos que o subgrupo A, que avaliou 20 imagens seguindo estritamente as orientações contidas no manuscrito original americano ${ }^{15}$, não reconheceu nem descreveu as imagens com conteúdo emocional adequadamente $(\hat{\mathrm{A}}<0,05)$ quando comparado ao subgrupo B, que reconheceu e descreveu mais de $75 \%$ das imagens com conteúdo emocional com acurácia após as modificações realizadas no manual, como, por exemplo, tamanho da imagem projetada, tempo de projeção da imagem, luminosidade das salas, etc. Essas modificações podem ser encontradas descritas na adaptação do Manual para Aplicação do IAPS para Idosos no Brasil, disponível em www.unifesp.br/ dpsicobio/adap/adapta.htm.

Já na segunda etapa, realizadas as devidas modificações no manual, submetemos os mesmos 48 idosos à avaliação dos demais 71 novos diapositivos oriundos do IAPS seguindo a classificação emocional do SAM. Estes escores do SAM concernentes ao nível de alerta e valência afetiva são apresentados na Tabela 1 .

A comparação das médias alcançadas relativas à valência afetiva (Tabela 2) entre a amostra de idosos brasileiros e jovens brasileiros e americanos não apresentou diferença estatisticamente significante - teste 
Tabela 1 - Escores Self Assessment Manikin para os 71 diapositivos estudados por uma amostra de idosos da Universidade Aberta da Terceira Idade (Universidade Federal de São Paulo), relativos ao nível de alerta e valência afetiva

\begin{tabular}{|c|c|c|c|c|c|c|c|c|c|}
\hline \multirow[b]{2}{*}{ IAPS } & \multicolumn{2}{|c|}{ Valência } & \multicolumn{2}{|c|}{ Alerta } & \multirow[b]{2}{*}{ IAPS } & \multicolumn{2}{|c|}{ Valência } & \multicolumn{2}{|c|}{ Alerta } \\
\hline & Média & DP & Média & DP & & Média & DP & Média & DP \\
\hline 2.040 & 8,02 & 2,52 & 2,81 & 3,09 & 8.490 & 7,08 & 2,34 & 5,08 & 3,21 \\
\hline 6.410 & 1,60 & 1,38 & 7,23 & 2,82 & 3.400 & 1,58 & 1,71 & 7,85 & 2,54 \\
\hline 4.500 & 5,85 & 3,70 & 2,98 & 3,09 & 6.560 & 1,13 & 0,61 & 8,27 & 2,24 \\
\hline 1.500 & 6,77 & 3,08 & 4,52 & 3,30 & 9.320 & 1,48 & 1,09 & 6,83 & 2,98 \\
\hline 3.120 & 1,44 & 1,54 & 7,29 & 3,05 & 3.500 & 1,04 & 0,62 & 8,00 & 2,53 \\
\hline 1.610 & 8,27 & 1,93 & 2,13 & 2,13 & 9.230 & 1,98 & 2,02 & 7,50 & 3,46 \\
\hline 5.750 & 8,02 & 2,44 & 1,88 & 2,15 & 2.490 & 2,83 & 2,26 & 5,83 & 2,91 \\
\hline 6.200 & 1,54 & 1,79 & 7,65 & 3,01 & 6.370 & 1,13 & 0,73 & 7,85 & 2,58 \\
\hline 3.030 & 1,50 & 1,88 & 7,17 & 3,06 & 8.501 & 7,90 & 2,27 & 3,25 & 3,15 \\
\hline 5.520 & 6,48 & 2,41 & 3,33 & 2,60 & 9.520 & 4,40 & 2,86 & 4,90 & 3,05 \\
\hline 2.030 & 7,69 & 2,81 & 2,69 & 2,91 & 6.570 & 1,15 & 0,62 & 7,90 & 2,77 \\
\hline 3.140 & 1,27 & 1,32 & 7,52 & 2,87 & 7.430 & 7,38 & 2,53 & 3,40 & 2,93 \\
\hline 8.090 & 7,42 & 2,35 & 3,50 & 2,76 & 4.700 & 8,23 & 1,56 & 2,25 & 2,07 \\
\hline 7.230 & 7,85 & 2,32 & 3,46 & 3,03 & 3.071 & 1,33 & 1,33 & 7,63 & 2,77 \\
\hline 7.500 & 6,77 & 2,26 & 3,69 & 2,58 & 8.600 & 6,10 & 2,82 & 3,60 & 2,54 \\
\hline 1.090 & 2,25 & 2,38 & 7,10 & 3,08 & 6.510 & 1,21 & 0,74 & 8,00 & 2,52 \\
\hline 4.650 & 7,15 & 2,24 & 3,25 & 2,73 & 3.550 & 1,15 & 0,62 & 7,90 & 2,76 \\
\hline 2.340 & 7,63 & 3,15 & 1,88 & 2,58 & 5.470 & 7,06 & 2,42 & 3,98 & 2,54 \\
\hline 3.170 & 0,96 & 0,54 & 7,31 & 3,22 & 6.540 & 1,40 & 1,59 & 7,85 & 2,76 \\
\hline 2.520 & 3,83 & 2,90 & 5,21 & 3,33 & 7.480 & 7,44 & 2,53 & 3,04 & 2,80 \\
\hline 9.270 & 4,52 & 2,87 & 5,29 & 2,90 & 5.982 & 7,52 & 2,45 & 2,08 & 2,12 \\
\hline 2.160 & 7,29 & 2,74 & 3,06 & 2,64 & 1.111 & 1,50 & 1,43 & 7,19 & 3,14 \\
\hline 7.190 & 5,21 & 2,95 & 5,00 & 2,85 & 5.532 & 5,77 & 2,73 & 3,77 & 2,70 \\
\hline 5.870 & 7,27 & 2,68 & 2,21 & 2,51 & 4.004 & 6,60 & 2,94 & 3,06 & 2,59 \\
\hline 3.230 & 1,85 & 1,69 & 7,10 & 2,71 & 9.470 & 1,98 & 2,10 & 7,02 & 2,98 \\
\hline 4.770 & 6,83 & 2,64 & 3,52 & 3,00 & 9.621 & 3,65 & 2,98 & 5,23 & 3,30 \\
\hline 7.580 & 8,04 & 1,91 & 2,46 & 2,44 & 8.161 & 7,19 & 2,56 & 3,67 & 2,82 \\
\hline 1.280 & 1,79 & 1,71 & 6,79 & 2,86 & 1.302 & 2,40 & 2,35 & 7,67 & 2,56 \\
\hline 2.710 & 1,98 & 1,86 & 7,17 & 2,68 & 1.201 & 1,27 & 0,98 & 8,15 & 1,99 \\
\hline 9.300 & 1,40 & 1,38 & 7,23 & 3,09 & 2.092 & 7,54 & 2,58 & 2,33 & 2,30 \\
\hline 2.800 & 1,65 & 1,36 & 8,08 & 2,01 & 4.609 & 7,71 & 2,47 & 2,52 & 2,35 \\
\hline 1.930 & 1,96 & 1,88 & 7,50 & 2,47 & 7.224 & 4,92 & 2,34 & 4,29 & 2,53 \\
\hline 8.480 & 2,08 & 2,06 & 7,21 & 2,82 & 7.285 & 6,88 & 2,46 & 3,31 & 2,56 \\
\hline 9.400 & 1,71 & 1,79 & 7,50 & 2,52 & 9.182 & 2,92 & 2,72 & 5,44 & 2,96 \\
\hline 8.510 & 7,98 & 2,15 & 2,21 & 2,00 & 9.921 & 1,81 & 2,03 & 7,46 & 3,07 \\
\hline 9.290 & 1,96 & 1,84 & 6,31 & 3,07 & & & & & \\
\hline
\end{tabular}

Tabela 2 - Estatística descritiva 1: escores de valência afetiva nas três amostras estudadas

\begin{tabular}{lcccccc}
\hline Valência & $\mathbf{n}$ & Média & $\mathbf{s}^{\mathbf{2}}$ & $\mathbf{s}$ & Kolmogorov-Smirnov & Intervalo \\
\hline UATI & 71 & 4,35 & 7,66 & 2,77 & $<0,01$ & $1,46-8,17$ \\
Jovens americanos & 71 & 4,58 & 4,57 & 2,14 & $<0,15$ & $1,46-8,17$ \\
Jovens brasileiros & 71 & 4,40 & 7,54 & 2,75 & $<0,05$ & $1,00-8,93$ \\
\hline
\end{tabular}

UATI $=$ Universidade Aberta da Terceira Idade.

de Kruskal-Wallis $\Rightarrow \mathrm{H}(2, \mathrm{~N}=213)=1,331$; $p=0,5140$. No entanto, foi observada uma diferença estatística na comparação do nível de alerta entre os grupos - teste de Kruskal-Wallis $\Rightarrow \mathrm{H}(2, \mathrm{~N}=213)=$ $11,02 ; \mathrm{p}=0,004]-$, principalmente no que concerne à comparação de jovens brasileiros e idosos da UATI (Mann-Whitney $\Rightarrow U=1838 ; Z=-2,78 ; p=0,0054$ ) e jovens brasileiros e americanos (Mann-Whitney $\Rightarrow$ $\mathrm{U}=1802 ; \mathrm{Z}=-2,93 ; \mathrm{p}=0,0034)$. No entanto, quando se compara os idosos da UATI e os jovens americanos, essa diferença deixa de ser estatisticamente significante (Mann-Whitney $\Rightarrow U=2421 ; \quad Z=0,41$; $p=0,685)$. Estes resultados podem ser observados descritos na Tabela 3.

A Tabela 4, por sua vez, mostra os resultados da forte correlação negativa de Spearman $\left(r_{s}\right)$ entre o nível de alerta e de valência afetiva nas três amostras envolvidas. 
Tabela 3 - Estatística descritiva 2: escores de nível de alerta nas três amostras estudadas

\begin{tabular}{lcccccc}
\hline Alerta & $\mathbf{n}$ & Média & $\mathbf{s}^{\mathbf{2}}$ & $\mathbf{s}$ & Kolmogorov-Smirnov & Intervalo \\
\hline UATI & 71 & 5,29 & 4,75 & 2,18 & $<0,01$ & $1,88-8,27$ \\
Jovens americanos & 71 & 5,29 & 1,32 & 1,15 & $>0,20$ & $2,81-7,21$ \\
Jovens brasileiros & 71 & 6,16 & 4,1 & 2,02 & $<0,10$ & $2,18-8,93$ \\
\hline
\end{tabular}

UATI $=$ Universidade Aberta da Terceira Idade.

Tabela 4 - Correlação de Spearman $\left(\mathrm{r}_{\mathrm{s}}\right)$ entre nível de alerta e valência afetiva das três amostras estudadas

\begin{tabular}{lcc}
\hline Valência x alerta & Correlação de Spearman & $\mathbf{p}<\mathbf{0 , 0 1}$ \\
\hline UATI & $-0,930734$ & 0,00 \\
Jovens brasileiros & $-0,829466$ & 0,000000 \\
Jovens americanos & $-0,543609$ & 0,000001 \\
\hline
\end{tabular}

UATI $=$ Universidade Aberta da Terceira Idade.

Na Figura 1, é demonstrado um gráfico de dispersão (formado pelos eixos do nível de alerta e da valência afetiva e denominado espaço afetivo) obtido pela análise dos relatos afetivos subjetivos dos indivíduos idosos da UATI.

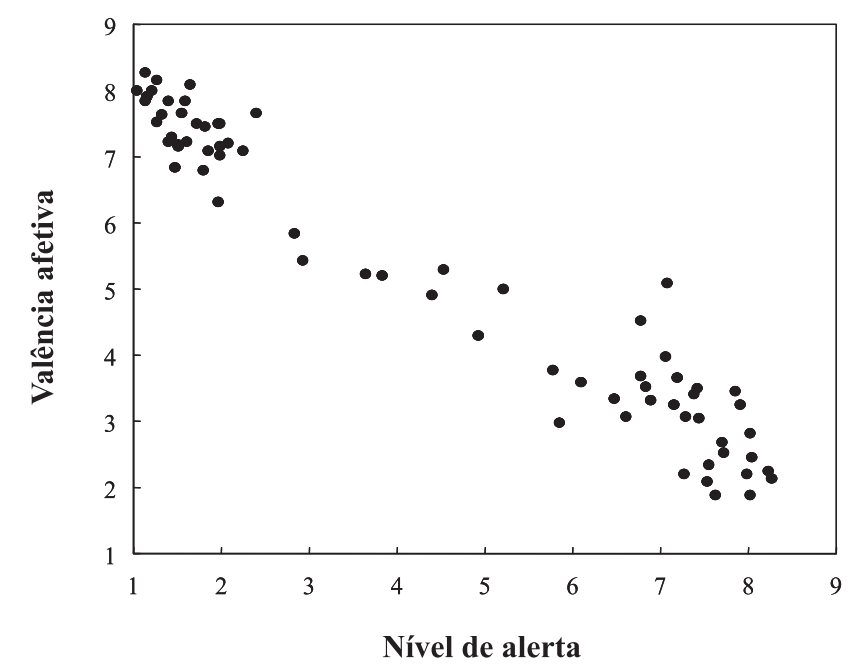

Figura 1 - Gráfico do espaço afetivo da correlação entre nível de alerta e valência afetiva obtida pela análise dos relatos afetivos subjetivos de uma amostra geriátrica da Universidade Aberta da

Terceira Idade - Universidade Federal de São Paulo

Finalmente, em um teste de freqüência $\left(\chi^{2}\right)$, é detectada uma diferença estatisticamente significante quanto ao nível de alerta suscitado para os idosos brasileiros em relação aos jovens brasileiros $\left(\chi^{2} \mathrm{p}=\right.$
0,01). O que é neutro para os idosos pode não sê-lo para os jovens. $\mathrm{O}$ mesmo não acontece quanto à valência afetiva $\left(\chi^{2} \mathrm{p}=0,07\right)$ nos dois grupos estudados. 


\section{Discussão}

O material aqui apresentado é resultado de um projeto-piloto, caracterizado como um fragmento de um estudo mais longo ${ }^{22}$, cujo objetivo básico é explorar como idosos normais avaliariam, através de relatos afetivos subjetivos, um volume de imagens carregadas de emoção oriundas do IAPS. Também é de grande interesse uma comparação entre esses relatos subjetivos dos indivíduos idosos com os feitos por jovens no mesmo período. Na ocasião, um estudo paralelo se desenvolveu com indivíduos jovens ${ }^{17} \mathrm{em}$ nosso departamento.

Como já citado, a grande maioria dos trabalhos feitos com as imagens oriundas do IAPS considera somente indivíduos jovens como objeto de estudo. $\mathrm{Na}$ literatura concernente a esses trabalhos, encontramos um gráfico denominado espaço afetivo (Figura 1) $)^{15-17}$, que é, em geral, bastante útil para a definição do padrão de avaliação afetiva de diferentes amostras. A figura do espaço afetivo obtida pelos idosos neste trabalho tem uma tendência à linearidade concentrada nos pólos, diferente dos resultados obtidos pelos jovens. A figura polarizada parece sugerir que as imagens desprazerosas alertariam enquanto as prazerosas relaxariam os idosos diante de imagens carregadas de teor emocional. Poderiam ainda sugerir que essas mesmas imagens tenderiam a provocar respostas afetivas do tipo "tudo ou nada", enfatizando extremos emocionais de valência afetiva e nível de alerta em detrimento de detalhes neutros. A forte correlação negativa entre o nível de desprazer e o grau de alerta deste estudo-piloto indica que há uma associação estatística entre essas variáveis. A viabilidade das conclusões acima pode ser corroborada por trabalhos similares, existentes na literatura, que sugerem que as diferenças na natureza das dimensões afetivas podem ser obtidas em função de variáveis como idade, psicopatia ou lesão cerebral. Sugerem ainda que os estímulos alertantes são avaliados como desprazerosos para os idosos e para os pacientes depressivos. Já as imagens alertantes prazerosas parecem não ser relevantes a essa categoria estudada ${ }^{11,23}$

Os resultados aqui obtidos encontram ainda guarida em mais alguns trabalhos que mostram que a avaliação emocional ganha valor privilegiado no diaa-dia do idoso ${ }^{5}$. De acordo com a literatura afim, os idosos tenderiam a enfatizar os traços emocionais de uma hipotética situação em detrimento de detalhes. Essa faixa etária tende a lembrar proporcionalmente mais informações com traços emocionais do que detalhes contextuais e espaciais em testes de evocação livre de memória, quando comparada com os jovens. Essa característica guarda íntima relação com a faixa etária. Quanto mais idoso o sujeito estudado, mais traços carregados de emoção serão lembrados. Portanto, faz sentido termos um gráfico do espaço afetivo do tipo "tudo ou nada" (Figura 1). As 71 imagens aqui consideradas migram em grande quantidade do estado neutro original observado em jovens para extremos afetivos subjetivos. As freqüências observadas quanto ao seu grau de alerta, no grupo idoso, são diferentes estatisticamente daquelas esperadas para os jovens. O mesmo não ocorre para a dimensão valência afetiva.

Assim, como idosos se beneficiariam biologicamente dessa forma diferente de perceber a emoção? As pesquisas indicam que os idosos parecem ter uma maior habilidade de regular suas emoções do que uma população de jovens. A estratégia utilizada por eles para alterar o impacto da resposta emocional seria focar em uma resposta antes de gerar tal emoção ${ }^{24}$. A regulação do bemestar emocional pode ser bem equilibrada entre adultos idosos e até melhor, em algumas situações, quando comparada à da população mais jovem ${ }^{25}$. A teoria da seletividade socioemocional (TSSE) diz que idosos dão mais valor a objetivos com significado emocional e investem mais cognitiva e comportamentalmente suas forças visando a esses alvos ${ }^{26}$. Assim, a emoção direcionaria processos cognitivos, e, ao direcioná-los, a memória formada poderia sofrer influência em seu conteúdo.

Os idosos seriam direcionados por avaliação de estímulos positivos ou negativos? Geralmente, estímulos prazerosos tendem a fazer a aproximação da fonte estimulante, e os desprazerosos, o distanciamento. Neste experimento, observamos que idosos trabalham em extremos de valência afetiva e nível de alerta. Porém, os que causam alerta e uma possível atividade física comportamental são os estímulos desprazerosos. As memórias, o aprendizado e o comportamento tendem, então, a ser melhores processados mediante a apresentação de um estímulo e/ou em situações que gerem estado de alerta ${ }^{27,28}$. Certos autores ${ }^{29}$ afirmam que não é o aspecto prazeroso ou desprazeroso de um estímulo que favorece a memória, mas o aspecto alertante desse estímulo. Portanto, é viável que as informações aprendidas e necessárias no dia-a-dia para manter a integridade corporal do idoso dependam do perfeito reconhecimento do que venha a ser desprazeroso. Quanto maior o alerta, maior a atenção e mais fiel será o traço de memória criado e aprendido. Nos idosos, o maior grau de alerta é alcançado por estímulos negativos, como vimos neste trabalho. Portanto, é possível que, em geriatria, as memórias e os comportamentos sejam guiados por uma avaliação acurada do aspecto desprazer, pois é esse aspecto que provoca uma resposta fisiológica alertante. Um forte 
foco emocional parece ser básico para uma avaliação cognitiva geriátrica.

Anatomicamente, as integridades dos complexos amigdalóides, bem como outras áreas límbicas adjacentes, talvez também estejam envolvidas nessa resposta "tudo ou nada" dos idosos e sejam necessárias na avaliação emocional ${ }^{30}$. Todavia, a literatura guarda certa controvérsia sobre o assunto. $\mathrm{O}$ envelhecimento do complexo amígdalo-hipocampal e entorrinal parece involuir em um ritmo desproporcional, quando comparado a outras regiões do cérebro, ou mesmo não mostrar sinais de involução ${ }^{24}$. Ou seja, existem evidências do envelhecimento em poupar seletivamente uma parte do sistema límbico e, em contrapartida, comprometer de uma forma importante os sistemas frontal dorso-lateral e órbito-frontal.

\section{Conclusões}

Em suma, o projeto-piloto aqui apresentado sustenta o anteriormente sugerido na literatura: a forma com que os indivíduos idosos percebem e relatam imagens carregadas de emoção pode ser diferente daquela apresentada por sujeitos jovens, principalmente no que diz respeito a estímulos emocionais alertantes. Essa constatação aponta para a possibilidade de uma tendência a considerar uma emoção mais intensamente do que os jovens, relegando neutralidade aos poucos estímulos do ambiente e corroborando, assim, o aspecto "tudo ou nada" observado no gráfico do espaço afetivo dessa amostra em especial. O que é neutro para os jovens pode não sê-lo para os idosos. Portanto, uma definição do que seja prazeroso, desprazeroso e neutro, bem como, e principalmente, do que causa alerta e relaxamento em uma população idosa deve ser bem esclarecida antes de se avaliar a emoção e a atenção e acessar a memória emocional nesse grupo. Uma normatização dos instrumentos IAPS/SAM é condição essencial para isso.

Devemos também considerar que os resultados acima relatados esbarram fortemente nas suas limitações estatísticas. Aqui fica claro que se trata de uma amostra pequena, carregada da desproporção de gênero e restrita a uma região nacional específica. Todavia, sabemos que uma normatização nacional para idosos se faz urgentemente necessária, o que adiantamos já estar sendo providenciado.

\section{Referências}

1. Pickholtz JL, Malamut BL. Cognitive changes associated with normal aging. In: Sirven JI, Malamut BL. Clinical neurology of the older adult. Philadelphia: Lippincott Williams \& Wilkins; 2002. p. 56-64.

2. Denburg NL, Buchanan TW, Tranel D, Adolphs R. Evidence for preserve emotional memory in normal elderly persons. Emotion. 2003;3(3):239-54.

3. Mather M, Johnson MK. Choice-supportive source monitoring: Do our decisions seem better to us as we age? Psychol Aging. 2000;15(4):596-606.

4. Mather M, Carstensen LL. Aging and attentional biases for emotional faces. Psychol Sci. 2003;14(5):409-15.

5. Charles ST, Mather M, Carstensen LL. Aging and emotional memory: the forgettable nature of negative images for older adults. J Exp Psychol Gen. 2003;132(2):310-24.

6. Keefover RW. Aging and cognition. Neurol Clin. 1998;16(3):63548 .

7. Mather M, Johnson MK. Affective review and schema reliance in memory in older and younger adults. Am J Psychol. 2003;116(2):169-89.

8. Mather M, Canli T, English T, Whitfield S, Wais P, Ochsner K, et al. Amygdala responses to emotionally valenced stimuli in older and younger adults. Psychol Sci. 2004;15(4):259-63.

9. Bradley MM, Lang PJ. Measuring emotion: Behavior, feeling and physiology. In: Lane R, Nadel L, eds. Congitive neuroscience of emotion. New York: Oxford University; 2000. p. 242-73.

10. Bradley MM, Greenwald MK, Petry MC, Lang PJ. Remembering pictures: pleasure and arousal in memory. J Exp Psychol Learn Mem Cogn. 1992;18(2):379-90.

11. Bradley MM, Lang PJ. Measuring emotion: the self-assessment manikin and the semantic differential. J Behav Ther Exp Psychiatry. 1994;25(1):49-59.

12. Cook EW 3rd, Melamed BG, Cuthbert BN, McNeil DW, Lang PJ. Emotional imagery and the differential diagnosis of anxiety. J Consult Clin Psychol. 1988;56(5):734-40.

13. Baddeley AD. Human memory: theory and practice. Allyn \& Bacon; 1998. p. 273-92.

14. Cahill L. Neurobiology of memory for emotional events: converging evidence from ingra-human and human studies. Cold Spring Harb Symp Quant Biol. 1996;61:259-64.

15. Lang PJ, Bradley MM, Cuthbert BN. International Affective Picture System (IAPS): Instruction Manual and Affective Ratings. Gainesville: University of Florida; 1999.

16. Moltó J, Montañés S, Poy R, Segarra P, Pastor MC, Tormo MP, et al. Un nuevo método para el estudio experimental de las emociones: el internacional affective picture system (IAPS). Adaptación española. Rev Psicol Gen Aplicada. 1999;52(1):5587.

17. Ribeiro RL. Avaliação de relatos subjetivos e de alterações fisiológicas a estímulos do "International Affective Picture System" (IAPS) em estudantes universitários brasileiros (tese). São Paulo: Universidade Federal de São Paulo, Escola Paulista de Medicina; 2003.

18. Lawton MP, Brody EM. Assessment of older people: selfmaintaining and instrumental activities of daily living. Gerontologist. 1969;9(3):179-86.

19. Hachinski VC, Iliff LD, Zilhka E, Du Boulay GH, McAllister VL, Marshall J, et al. Cerebral blood flow in dementia. Arch Neurol. 1975;32(9):632-7.

20. Freedman M, Leach L, Kaplan EF, Winocour G, Shulman KI, Deli DC. Clock drawing: a neuropsychological analysis. New York: Oxford University; 1994.

21. Okamoto IH. Aspectos cognitivos da doença de Alzheimer no teste do relógio: Avaliação de amostra da população brasileira (tese). São Paulo: Universidade Federal de São Paulo, Escola Paulista de Medicina; 2001.

22. Porto WG. Normatization of 702 images set from the International Affective Picture System (IAPS) in a Brazilian elderly sample for emotion and attention testing. Arq Neuro-Psiquiatr. 2006;64(1):162-3

23. Cuthbert BN, Bradley MM, Lang PJ. Psychophysiological responses to affective slides across the life span. Psychophysiology. 1988;25(4):441.

24. Mather M. Aging and emotional memory. Reisberg D, Hertel P, 
eds. Memory and emotion. New York: Oxford University. 2003. p. 272-307.

25. Carstensen LL, Charles ST. Emotion in the second half of life. Cur Directions Psychol Sci. 1998;7(5):144-49.

26. Charles ST, Mather M, Carstensen LL. Aging and emotional memory: the forgettable nature of negative images for older adults. J Exp Psychol Gen. 2003;132(2):310-24.

27. Izquierdo I. Memória. Porto Alegre: Artmed. 2002. p. 9-95.
28. LeDoux J. O cérebro emotional. Santos TB. Rio de Janeiro: Objetiva. 1998. p. 9-332.

29. Bradley MM, Greenwald MK, Petry MC, Lang PJ. Remembering pictures: pleasure and arousal in memory. J Exp Psychol. 1992;18(2):379-90.

30. Bradley MM, Lang PJ. Measuring emotion: the self-assessment manikin and the semantic differential. J Behav Ther Exp Psychiatry. 1994;25(1):49-59. 